\title{
Research on the Design of Experiential Commercial Space
}

\begin{abstract}
Siqun $\mathrm{Xie}^{1, *}$
${ }^{1}$ Shanghai Academy of Fine Arts, Shanghai University, Shanghai 200040, China

"Corresponding author. Email: xiesiqun@qq.com

ABSTRACT

In the era of Experience Economy, consumers' functional demand for commercial space has been upgraded from the traditional commodity display to meeting the needs of personalized experience. In the process of commercial space consumption, consumers are no longer satisfied with the traditional single material needs; instead, they pay more attention to the realization of their own experience process and spiritual needs in the commercial space. This paper takes the interior design of experiential commercial space as the research object, combining with the Experience EEI (Expectation, Event, Impact) Model proposed by Professor Xin Xiangyang, elaborates the personalized needs of consumers for commercial space experience under the Experience Economy, and puts forward the space design method of experiential commerce, which provides innovative design ideas for experiential commercial space design.
\end{abstract}

Keywords: Experience Economy, space design, interaction, self-creation, digitalization

\section{INTRODUCTION}

Experience Economy is the fourth type of economy ("Fig. 1") after Agricultural Economy, Industrial Economy and Service Economy, which is the extension of service economy and the inevitable result of the development of economic model. Experience Economy pursues the degree of consumers' perceptual satisfaction and pays attention to the self-experience in the process of consumption. Experience Economy starts from life and situation, shapes sensory experience and thinking identity, so as to seize consumers' attention, change consumption behavior, and find new survival value for commodities. Experience Economy is a new economic form, which shows the direction of economic and social development. Experience Economy gestates the significant changes of consumption mode and production mode, adapts to the speed of Experience Economy will become the key to the success or failure of future business competition.

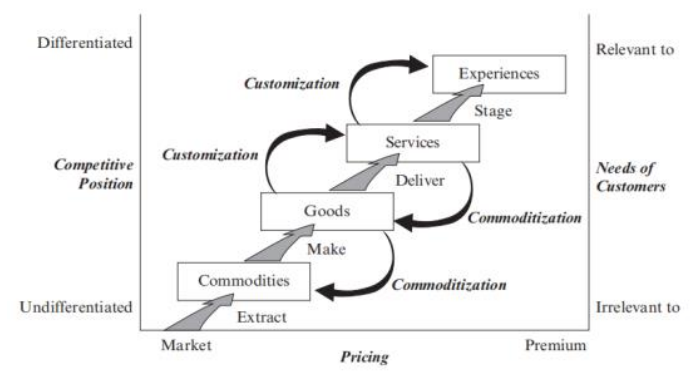

Fig. 1. Stage illustration of economic development (Source: Pine and Gilmore, 2011, p, 111).

Experience Economy takes "service" as "stage" and "commodity" as "prop" to integrate consumers into the stage of social evolution. In the era of Experience Economy, consumers have transformed from the traditional single material needs into their own spiritual pursuit. In 2001, Professor Dai Huisi of the Department of Sociology of Yale University pointed out in the publication of Consumer Culture and Consumer Revolution that if the 19th century was the century in which producers created the world, then the 20th century was the century in which consumers created the world. Entering the information age, the speed of information dissemination makes the current market more transparent, and consumers continue to occupy the dominant position of the market. In order to respond to the current and future market changes more positively, it is an inevitable trend for the future development of commercial space design to create 
experiential commercial space and meet the personalized consumption experience environment of consumers based on the experience process of consumers. ${ }^{1}$

\section{EXPERIENTIAL COMMERCE AND ITS CHARACTERISTICS}

With the development of e-commerce, the retail position of real estate is constantly losing. At the same time, the intensive construction of commercial real estate projects also leads to fierce competition in real business, and the traditional commercial space and business format no longer have advantages in attracting passenger flow. Against the background of Experience Economy, Experience Economy provides the direction of innovation and development for the design of physical commercial space. From then on, the traditional functional display design has been transformed into experience-oriented personalized space design to meet the design goals of consumers' self-realization in commercial space.

Experiential commerce is different from traditional commerce, which is a combination of retail-based formats. Experiential commerce emphasizes more on the participation, experience and feeling of consumers, and requires higher space and environment. The emergence of "experiential style" comes not only from the ever-upgrading commercial retail industry in the era, but also from the diversified needs and experience modes of consumers for commercial space.

The most common carrier of experiential commerce is shopping malls. In the carrier of traditional commercial space, there is no emphasis on "experience", only on whether the function of goods can meet the needs of consumers. Therefore, the format of commercial space does not have the characteristics of integrity and systematicness. Nowadays, with the concept of "experiential commerce" put forward, the perspective of space design has changed from traditional functional space design to consumeroriented experience process design, focusing on the richness and diversity of business combination.

Experiential commerce has the typical characteristics of emphasizing customers' psychological experience and three-dimensional feeling in the process of shopping. In order to meet the needs of consumers for experiential commercial space, against the background of Experience Economy, the traditional commodity display space design is transformed into experiential commercial space design, and the single display function of traditional commercial space is transformed into diversified experience function.

\footnotetext{
Dai Huisi, LU Hanlong. Consumer Culture and Consumer Revolution [J]. Sociological Research, 2001 (5): 117-125.
}

\section{THREE EXPERIENCING EEI MODEL IN THE DESIGN OF EXPERIENTIAL COMMERCIAL SPACE}

The Experience EEI Model establishes the theoretical framework of experience process for experience design. Experience EEI Model is an experience model proposed by Professor Xin Xiangyang in his article "From User Experience to Experience Design". The Experience EEI Model ("Fig. $2 ")$ treats Expectation, Event, and Impact as integral parts of an experience. On the one hand, expectation triggers and guides the beginning of events, and affects the process of events as participants are brought into the events for self-creation, thus becoming an important cause of understanding events, precipitating memories and discovering meanings; On the other hand, only when the event precipitates into memory and develops into a story worth sharing, can the construction of experience be truly completed and a special experience be concluded. Where events emphasize the importance of environmental conditions in the process of events, as well as the existence of self-creation activities of participants and their impact on the process. ${ }^{2}$

\footnotetext{
2 Xin Xiangyang. From user experience to experience design [J]. Packaging Engineering, 2019 (8): 60-67.
} 


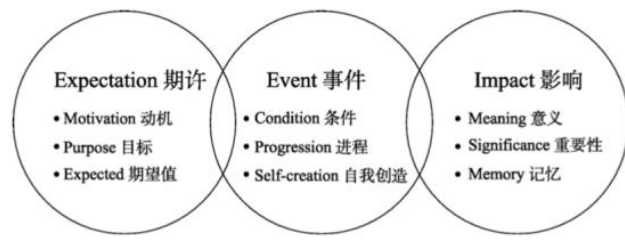

Fig. 2. The EEI Model of Experience (Source: From User Experience to Experience Design, Xin Xiangyang)

Because of the uniqueness of its process and result, every complete experience is an individual worthy of attention, a dynamic organization with a complete structure of "beginning, development and completion" under the guidance of a specific purpose. ${ }^{3}$ According to the positive degree of consumer participation in experience activities, consumer behavior can be divided into perception type and co-creation type. Perceptual behavior refers to consumers' physiological perception caused by some factors in the space, and the changes brought by perception occur in the hearts of customers, which does not affect the experience creation of the space, such as independent viewing, thinking and browsing commodities; Co-creative behavior refers to consumers' personal participation in the experience process and their personal influence on the experience activities, such as consulting commodity information, discussing with the surrounding customers, evaluating services, etc. The interactive space elements also have the ornamental attribute of display function when its empty, because of the interaction caused by consumers' fetching and browsing behavior at some time. The consumers who browse the goods are not completely isolated from the experience activities, and they also belong to the visual and auditory elements of other people's co-creative experience behavior. ${ }^{4}$

According to the space functional needs of experiential commerce and the experience needs of consumers, the space is divided into display space and interactive space. The exhibition space pays attention to the display and design of commodities, and establishes the experience tone for the space through the basic interactive design or interactive form display. Interactive space emphasizes the interactive experience process between people and space or people and commodities through interactive devices or service space. Display space should be designed as a complete comfortable space, must have a commodity space, service space and customer space in three parts. In the entire display space should be mobilized to cooperate with all the factors, so that the entire display space and Books, 1980.

DEWEY J. Art as Experience [M]. New York: Perigee

Chen Feihu. Looking at the Innovation of Contemporary Commercial Space Design from the Experience of Consumption Culture [J]. Journal of Hunan University (Social Sciences Edition), 2020 (5): 146-152. display process integrity, to provide consumers with a space to enjoy.

The positive degree of consumers' participation in experience activities in commercial space depends on whether the internal design of space can give consumers perception factors or guiding factors. Experiential commercial space division and consumer behavior are closely linked and interact with each other, forming one of the criteria to judge the success of experiential commercial space design. The theory of Experience Economy has a strong guiding significance for the design and operation of mainstream commercial buildings. In the book Brand Architecture-Architectural Design in the Experience Economy, Anna Collinman believes that architecture must be designed according to the acceptance and perception of users, so as to bring them the enjoyment of visual beauty and stimulate emotional resonance in the use process. ${ }^{5}$

The author will analyze the content and form of the existing experiential commercial space design through the Experience EEI Model, and try to analyze the experience design process that can enable consumers to consume in the experiential commercial space. Starting from the display form of experience in space, combined with the experience process of consumers, this paper puts forward innovative experience design, which provides inspiration and reference for the space design of experiential business in order to meet the diversified experience needs of future consumers.

\section{INNOVATION OF EXPERIENTIAL COMMERCIAL SPACE DESIGN IN THE ERA OF EXPERIENTIAL ECONOMY}

The rapid development of science and technology, as a catalyst in the electronic information age, makes offline business development pay more attention to the experience of consumers in the commercial space. Therefore, experiential commercial space design has gradually become the key to the transformation of traditional commercial space design. In order to meet the needs of consumers in the experience market, combined with the Experience EEI Model proposed above, this paper puts forward an innovative design method for experiential commercial space design.

Klingmann Anna. Brand Architecture [M]. Beijing: Publishing House of Electronics Industry, 2014:304. 


\section{A. Expectation: innovation of multi-dimensional sensory experience and construction of emotional experience mode}

The consumer behavior in the commercial space is closely related to the interactive experience and personal feelings in the commercial space. Before entering the commercial space, consumers will have consumption purposes, consumption motives, and subjective expectations generated by psychology. According to the experience and feelings of consumers, the interactive experience can be divided into three levels: the shallow sensory stimulation experience that can attract the attention of users, the immersion physical and mental interaction experience that can make users feel the immersion through space design, and the deep immersion interactive experience that can cause consumers to identify with values in space.

Experiential commercial space design will directly guide consumers to produce sensory stimulation from four aspects of vision, hearing, smell and touch. Sensory stimulation can make consumers produce physiological reactions, stimulate psychological reactions through physiological reactions, and evolve to the middle level of physical and mental interaction experience, thus slowly entering the deep level of immersion experience environment. This progressive relationship not only provides an objective design direction for the interactive experience mode in experiential commercial space design, but also makes it an entrance to establish consumers' emotional perception, and then constructs a stable and long-term emotional experience and emotional identity between consumers and commercial space.

Multi-dimensional sensory experience in space design pay more attention to the transmission of visual, auditory guidance and tactile interaction. Visual communication must be based on the flexible use and combination of brand system in space. Color, image, image, text, lighting is the visual space in the design of the key elements in the design of space must follow the unity, in order to provide a visual basis for the later interactive experience design. Located in Beijing Wangfujing shopping and entertainment business circle, the first LEGO brand flagship store ("Fig. 3") uses the color design of LEGO brand visual system to show the space color with Chinese traditional culture charm. The space top combined with the classic LEGO building blocks to design the top chandelier directly conveys the brand characteristics, and intuitively stimulates consumers' experience senses. In the commercial space design of LEGO flagship store, red, yellow and blue colors are used to construct the color system of LEGO commercial space, define people's visual impression, and then form consumers' emotional recognition of the brand space color.

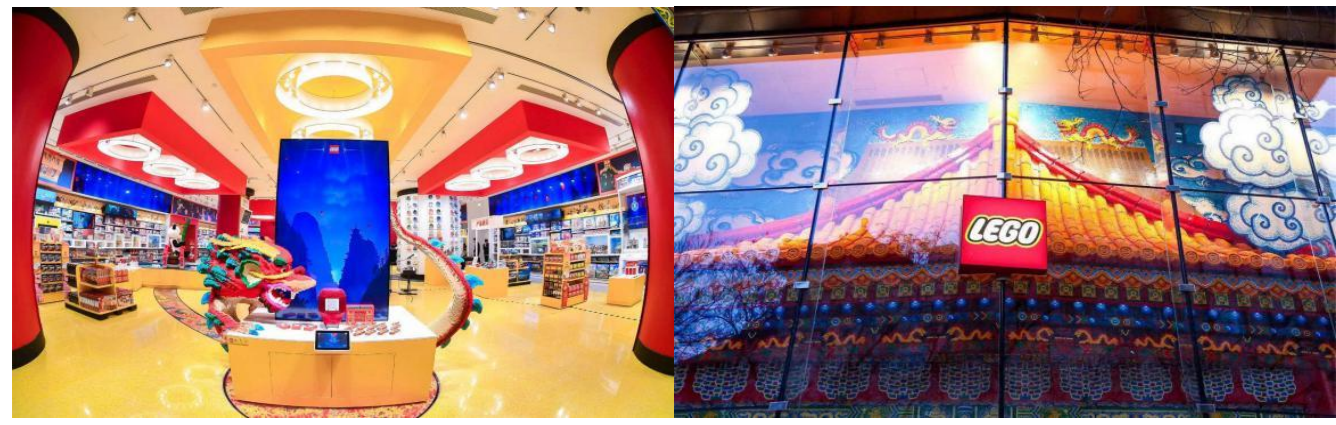

Fig. 3. Beijing Wangfujing Lego Flagship Store (Source: Network).

As an important factor affecting consumers' senses, hearing can guide consumers to interact and create a positive space atmosphere that meets the expectations of both sides. Auditory perception can evolve into emotional perception, and establish emotional feelings and emotional environment that meet brand expectations for consumers. As a grocery store brand, MUJI has established a new image of grocery store for people. The space division in the store can satisfy consumers to choose and buy comfortably and experience the experience process of goods freely. The selection of music in the store also fully demonstrates the positioning and functional design of MUJI brand, filling the whole commercial space with natural music as the background, and establishing the experience of pursuing natural shopping environment for consumers.
No matter how many times consumers enter the store, they will feel the transmission of nature-oriented brand design concept in MUJI store space as they enter the store for the first time.

Smell is more sensitive than other senses and has persistence. In the design of commercial space for consumers to create a personalized olfactory experience, can make consumers on the commercial space to form a long time unique impression. The most representative dim sum in Shanghai is butterfly cake, which is also the first choice for Chinese and foreign tourists as a companion gift. The Shanghai International Hotel sells butterfly cakes, one of its main products, and queues are endless every day. This is mainly due to its unique taste, which can give people a unique 
olfactory memory. Every time they pass around the store, they can smell the milk fragrance of the brand's baked butterfly crisp, and quickly form people's olfactory recognition of the butterfly crisp products sold by international hotels. Whenever you see butterfly cake, the first impression is not the inherent shape, but can find unique olfactory memory in many butterfly cake brand sales stores, and then rise to brand recognition.

Touch is the real presentation of the essential attributes, and it is also the primary sense of sensory experience. In experiential commercial design, the tactile experience of consumers mainly comes from displaying goods, decorative materials or space materials, etc. Products or materials with different material characteristics can convey different information and characteristics. Apple Experience Store ("Fig. 4") has been following the minimalist style, highlighting product experience and product services, which is one of the representative design cases of experiential business space design. Entering the Apple Experience Store, consumers can interact directly with the products and feel the charm of Apple products through intuitive touch experience. Apple store design follows the green design and environmental protection design, store display area selected materials are wood materials, compared with traditional materials, not only can prolong life, but also can convey brand design concept and design value.

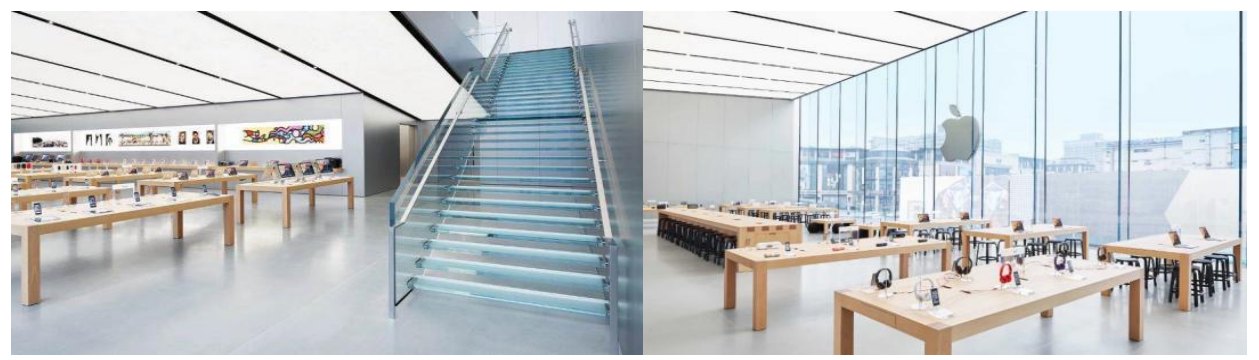

Fig. 4. Hangzhou Apple Experience Store (Source: Network).

\section{B. Event: personalizing the information transmission process and building a sustainable experience design}

The receiving port of information transmission in the commercial space is the consumer, and the transmitting port is the exhibition content in the commercial space. The exhibition design in the space will focus on the transmission and sustainability of information transmission. Information transmission has the characteristics of active communication at the transmission level, and consumers can choose to accept it according to their own needs. The sustainability of information transmission fully reflects the innovative form of experiential commercial space design, which can invite customers to create experience in commercial space, create personalized experience mode together, and establish a sustainable development experience model or experience process between commercial space and consumers.

Under the Experience Economy, the information content in the commercial space is not limited to the seller's unilateral will, but the material and emotion exchanged under the common needs and wishes of the seller and consumers. If the seller really wants to provide information to consumers and complete the transmission, the transmission activities must play an active role in the minds and bodies of consumers.
Artificial intelligence technology can be used to maximize the efficiency of information transmission. ${ }^{6}$

If we want to design a personalized information transmission process, we must establish a transmission mode with interactive attributes, which can provide effective transmission path and interesting interaction process for both sides. In the Experience Economy, in order to effectively achieve the above points, the intervention of science and art makes the experiential commercial space design innovative. The intelligent screen with intelligent interactive system is chosen to replace the traditional giant poster to complete the dynamic design and transmission of information transmission. Components in interactive systems and complex information systems can automatically identify and judge, help users identify and judge core information, ensure the transitivity of effective information, and optimize consumer experience.

Traditional retail stores have been unable to meet the needs of consumers. Under the Experience Economy, consumers want more, more fun and richer experience forms. In experiential commercial space design, the involvement of digital design increases the dynamic elements of interactive forms in space, and

\footnotetext{
6 Chen Feihu. Looking at the Innovation of Contemporary Commercial Space Design from the Experience of Consumption Culture [J], Journal of Hunan University (Social Sciences Edition), 2020 (5): 146-152.
} 
establishes digital information interaction and information dissemination channels for consumers. In the process of transmission, the seller inputs information to consumers, which can also be said to be the process of "education" by the seller to consumers. Liang Qichao put forward the idea of "interest education" on the issue of education, believing that "interest is the source of activities". Information transmission design in commercial space can be disseminated in the form of game mechanism or game, so that users can actively participate in information input, and then obtain users' main information. Shanghai 001, Nanjing East Road ("Fig. 5"), was originally Nike's first direct store in China, after upgrading and transformation, it has become Nike's first "House of Innovation" "in the world. The concept of "House of Innovation" "is an immersive experience store launched by Nike, hoping that consumers can experience Nike's cutting-edge innovation through entering the store, thus generating experience consumption and purchasing products. Digitalization is an important element of Nike Shanghai 001 ("Fig. 6"). In the design of experiential commercial space, the "core midfield" on B1 floor is the complete embodiment of digital display, no matter from which floor to enter the space, you can see a huge digital screen, which runs through the whole building from B1 to 3 floors. ${ }^{7}$ 


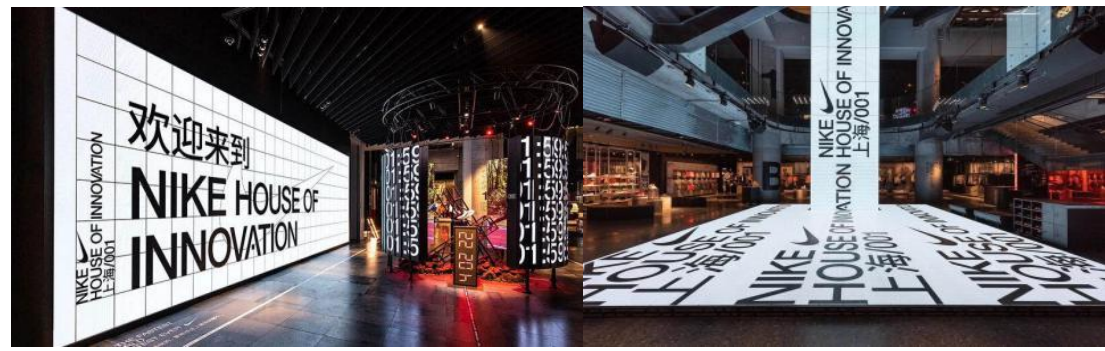

Fig. 5. Shanghai Nanjing East Road Nike 001 Store (Source: Network).

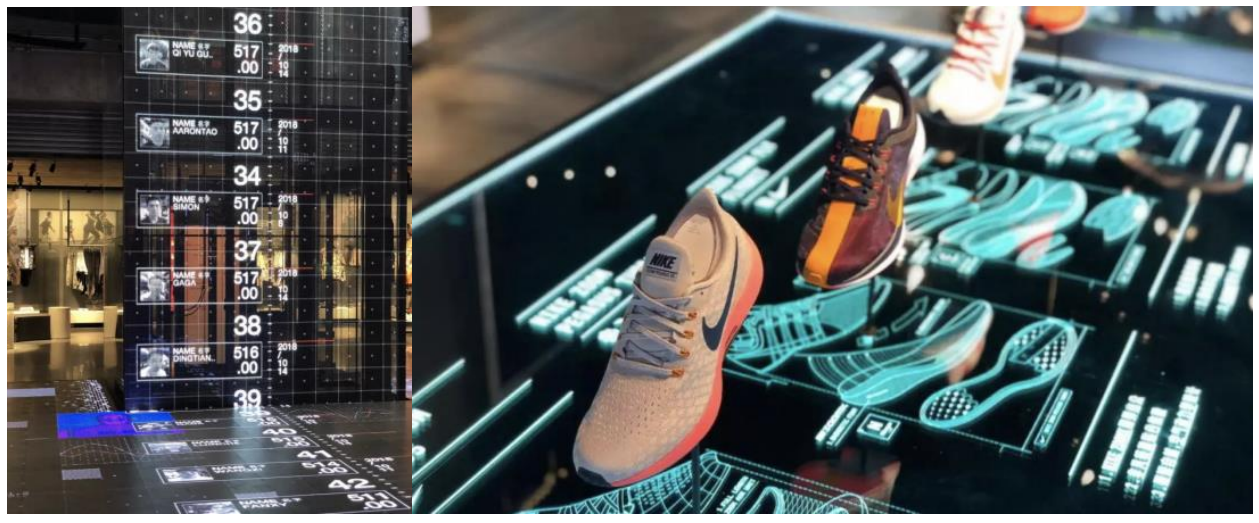

Fig. 6. Interactive device display of Nike 001 Store on Nanjing East Road, Shanghai (Source: Network).

The design principle of "Core Midfield" is adhering to the design principle of "interest is the source of activities", which fully reflects the "self-creation" in the Experience EEI Model. Game mechanism provides consumers with the motivation to experience actively. After consumers change into brand sports equipment, they can complete different sports challenges (such as touchdown jump, extreme trot, etc.) In the form of sports interaction under the guidance of intelligent terminals. In front of the experience area, the screen with flowing display results stimulates the user's desire to win or lose, so that the user can immerse himself in the experience. Real offline experience can make consumers more immersed in the function of products, stimulate users to experience consumption, and then establish the experience contact point between the internal experience of commercial space and the sustainable development of consumers, and ultimately transform into the quantitative goal of experience results.

\section{Influence: combining artistic expression and brand image to create a multi-dimensional aesthetic space of difference}

The experience behavior in a certain place is the time movement of the combination of static entity and dynamic virtual form of subjective people in objective space, starting with entering the place and ending with leaving the place, and the participation of place space throughout the whole experience process. Consumers enter the experiential business space, eager to get different from the daily life experience, get a new experience mode. To create experiential business space feelings usually take physical reduction or emotional remodeling two ways of expression to create can convey the aesthetic design as the core design can experience the commercial space form shaping the difference of the space image. ${ }^{8}$

Aesthetics is a kind of inner positive spirit and concept. The aesthetic experience proposed by Pyne is a kind of beautiful feeling produced by powerful attraction and appreciation. Like "perception", aesthetics has no standard definition and universal method of construction. To create an aesthetic feeling in the space of experiential merchants, two design expressions, physical restoration or emotional remodeling, are usually adopted. To create a communicative aesthetic design as the core, the design can experience the commercial space form, shape the spatial image of difference, and provide innovative ways to obtain competitive advantage. ${ }^{9}$

8 ZHENG Shu-zhen. Interior Design, Thinking and Method [M].Beijing: China Architecture \& Building Press, 2003.

9 Fan Jing. Consciousness Awakening of Art Appreciation [J].Grand View of Art, 2011 (4): 178-179. 
Most of the current space design has ignored the visual experience of consumers, not the real brand culture into the commercial space design, but a superficial visual effect to attract people's attention, in the long run, it has brought negative impact to the space content. Commercial space is a multi-dimensional interactive experience space. The interaction between people and goods, the interaction between people and space, and the interaction between space and goods all reflect the design opportunity points of experience and interaction.

Aesthetic image as a symbol of the spirit within the commercial space, must be combined with the corporate image or corporate characteristics, form a representative artistic image into the space design, and ultimately create a different multi-dimensional aesthetic space. Located in Shanghai's newest premier business and leisure complex, Societe Generale Taikoo Hui, Starbucks' ("Fig. 7") first fully immersive coffee experience in Asia, the bronze-roofed Shanghai Roastery looks like a giant coffee bean from the air. Starting from the innovation of experiential commercial space design, in order to enable customers to understand and experience coffee culture at close range, the design team combines with Chinese skilled craftsmen, starting with every detail of conception, layout and material, and combining with Chinese excellent traditional culture, presents a journey from a raw coffee bean to a cup of fragrant coffee. In this display process, for users, it is an aesthetic transmission with brand significance, more like an experience, a stage, or even a performance about aesthetics.

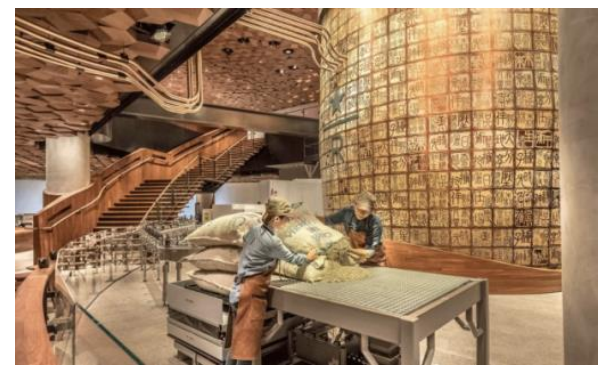

Fig. 7. Starbucks Shanghai Roastery in Shanghai (Source: Internet).

"The Yi Chuan Yi Zhuan" written by Cheng Yi in the Song Dynasty once had such a statement: "Astronomy is the principle of heaven; Humanity is the way of people." The refinement, amplification and embellishment of a particular culture will gradually become a kind of perspective, vigorous and full of strength. Starbucks full immersion coffee experience store space design reflects this very well, coffee bar almost all use deep walnut color interpretation, and coffee baking temperature change curve radian similar, its unique artistic beauty into the experience of commercial space design, constitute a brand of color aesthetics.

People's pursuit of beauty originates from material life and eventually rises to the spiritual level, which is the process of human pursuit of the soul. In the experience of commercial space design, pay attention to create differences in multi-dimensional aesthetic space, the brand image and artistic, practical combination, the formation of experiential commercial space memory, for the future of commercial space design is of great significance.

\section{CONCLUSION}

Compared with traditional commercial space design, experiential commercial space design under Experience Economy will pay more attention to the experience process and experience behavior of consumers. Faced with the current changing consumer groups, the progressive level of science and technology, combined with the Experience EEI Model process for experiential commercial space design innovation multidimensional sensory experience, build emotional experience mode; Establish the personalized information transmission process within the experiential commercial space, and provide opportunities for sustainable experience design; Based on experiential aesthetics, combined with artistic expression and brand image, multi-dimensional aesthetic space with differences is created. The traditional commercial space with display function will be transformed into interactive space with experienceoriented design, and efforts will be made to meet consumers' personalized experience needs in the experience era.

\section{References}

[1] Dai Huisi, LU Hanlong.Consumer Culture and Consumer Revolution [J].Sociological Research, 2001 (5): 117-125.

[2] Xin Xiangyang.From user experience to experience design [J].Packaging Engineering, 2019 (8): 60-67.

[3] DEWEY J. Art as Experience [M]. New York: Perigee Books, 1980

[4] Chen Feihu. Looking at the Innovation of Contemporary Commercial Space Design from the Experience of Consumption Culture $[\mathrm{J}]$, Journal of Hunan University (Social Sciences Edition), 2020 (5): 146-152.

[5] Klingmann Anna. Brand Architecture [M].Beijing: Publishing House of Electronics Industry, 2014:304.

[6] Chen Feihu. Looking at the Innovation of Contemporary Commercial Space Design from the Experience of Consumption Culture $[\mathrm{J}]$, Journal of Hunan University (Social Sciences Edition), 2020 (5): 146-152.

[7] Zhao Yongping. Liang Qichao's Discussion on Interest Education and Educational Interest and Its Enlightenment to Today's Education $[\mathrm{J}]$. Journal of Jiangsu Second Normal University, 2017 (2): 42-45.

[8] ZHENG Shu-zhen.Interior Design, Thinking and Method [M].Beijing: China Architecture \& Building Press, 2003.

[9] Fan Jing.Consciousness Awakening of Art Appreciation [J] Grand View of Art, 2011 (4): 178-179. 